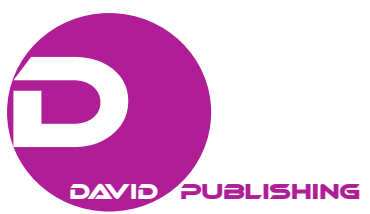

\title{
Perception of Fake News: A Survey of Post-Millennials
}

\author{
Niaz Ahmed \\ St. Cloud State University, Minnesota, USA
}

\begin{abstract}
This study examined post-millennials' news consumption habits and perception of fake news in social media. A survey was completed by a non-random sample of 415 students at State University of New York in Oneonta during the academic year 2017-2018. The results revealed that more than half of post-millennials accessed various social media several times a day, while nearly one in five admitted accessing social media every hour of the day. As for the amount of time devoted to social media, nearly one-third of the students admitted using social media for 7-10 hours per day, and slightly less than one-third of the students spent 5-6 hours per day on social media. With regard to news consumption habits of post-millennials, data analysis revealed that nine in 10 students used their smartphones to check the news online, and most students used multiple sources of news. About four-fifths of the students obtained their news from online newspapers and magazines, while three-fifths of them also used social media for obtaining news. As for the amount of time devoted to consuming news, four-fifths of the students indicated that they spent 1-2 hours in a typical day for news consumption. In terms of exposure to fake news, nine in 10 students indicated that they had seen some news on social media that turned out to be fake news. These findings may have significant implications for social media as they plan to counter the proliferation of fake news on their platforms.
\end{abstract}

Keywords: news consumption habits, perception of fake news, post-millennials

The advent of the smartphone and social media has significantly transformed our lives in terms of how we communicate and entertain ourselves. In recent years, smartphone and social media usage has grown exponentially among the general public and specifically within the post-millennials or Generation Z (Statista, 2015). The Generation $Z$ is the demographic cohort born during the mid-1990s to mid-2000s (roughly 13-24 years of age today) and has followed the millennials or Generation Y (Seemiller \& Grace, 2016; Wood, 2013). Generation $\mathrm{Z}$ is the first cohort to have smartphones, social media, and other technologies readily available at a young age.

Although a significant amount of research has been done in the recent past about the use of social media among Generations $\mathrm{X}$ and $\mathrm{Y}$, insufficient research exists about post-millennials' news consumption habit and perception of fake news as it relates to the social media. Since the digital world is changing rapidly every year, it is important to track its usage and impacts on an ongoing basis for detecting any trends and correlations, and for identifying significant implications. The present study attempts to contribute to the evolving knowledge by examining the implications of post-millennials' news consumption habits and perception of fake news.

Niaz Ahmed, Dr., Professor of Mass Communication, St. Cloud State University, Minnesota, USA. 


\section{Literature Review}

Most recent studies (Oberst, Wegmann, Stodt, Brand, \& Chamarro, 2017; Buglass, Binder, Betts, \& Underwood, 2017) have found that excessive use of social media was partially due to what has been called the fear of missing out (FOMO). Al-Menayes' 2016 study also revealed that FOMO was associated with social media addiction. Furthermore, a new phenomenon termed nomophobia (no mobile phone phobia) has been identified as a symptom characterized by the fear of being without one's mobile phone. Nomophobia traits include feelings of anxiety when the phone is not available, repeatedly checking of messages, hearing of nonexistent phantom ring tones, and preference for mobile interaction over face to face communication (Billieux, Maurage, Lopez-Fernandez, Kuss, \& Griffiths, 2015).

A 2009 survey of college students (Jarvis, Stroud, \& Gilliland) revealed that most students accessed news on the web and cable television, and do not usually trust the sources.

A 2015 study by Mitchell and Gottfried found that 61\% of millennials get their political news on Facebook, while $51 \%$ of Gen Xers get political news on Facebook. The results also indicated that about $26 \%$ of millennials chose politics and government as the topics they are most interested in.

A 2012 survey (Hermida, Fletcher, Korell., \& Logan) of 1600 Canadians revealed that two-fifths of social media users received news from people they follow on services like Facebook, while one-fifth got news from news media they follow. Rosengard, Tucker-McLaughlin, and Brown (2014) found that students, who are exposed to news on social media, often rely on other internet sources to confirm it. A 2016 study revealed that majority of college students get breaking news from Twitter initially, which leads them to get more information from websites of traditional news organizations (Tandoc \& Johnson, 2016).

\section{Methodology}

A survey of college students was conducted to examine the smartphone and social media usage and addiction among Generation Z during the academic year 2017-2018. The survey was administered via SurveyMonkey and was completed by a non-random sample of 415 students at State University of New York in Oneonta. This study poses the following research questions:

RQ 1: What is the nature of news consumption among post-millennials? Are there any statistically significant differences in terms of gender and age?

RQ 2: What are the perceptions of post-millennials about fake news on social media? Are there any statistically significant differences in terms of gender and age?

\section{Results}

The survey was completed by 415 students at State University of New York in Oneonta. The completion rate of the survey was $99 \%$. The response rate was very high due to the fact that the students received extra points for completing the survey as part of a class requirement. Approximately $56 \%$ of the respondents were females, and $43 \%$ were males. About $63 \%$ of the subjects were $18-20$ years of age, and $27 \%$ of them were 21-25. In terms of race, about $54 \%$ of the respondents were white and $46 \%$ were non-white (Hispanic, Black, Asian, Native American, Mixed). Approximately 48\% of the subjects were freshman and sophomores, $42 \%$ were juniors and seniors, and $10 \%$ were graduate students. 


\section{How Does Generation Z Use Social Media?}

The results revealed that Snapchat, Instagram, Facebook, and Twitter are the most widely used social media among Generation $\mathrm{Z}$ with majority of the respondents having multiple social media accounts. As shown in Table 1, 93\% of the students had an account on Facebook, 95\% had an account on Snapchat, 88\% had an account on Twitter, and 58\% had an account on Instagram. In addition, about 41\% used YouTube while 15\% used Google Plus.

Table 1

Most Frequently Used Social Media

Types of social media

Frequency and percent of respondents

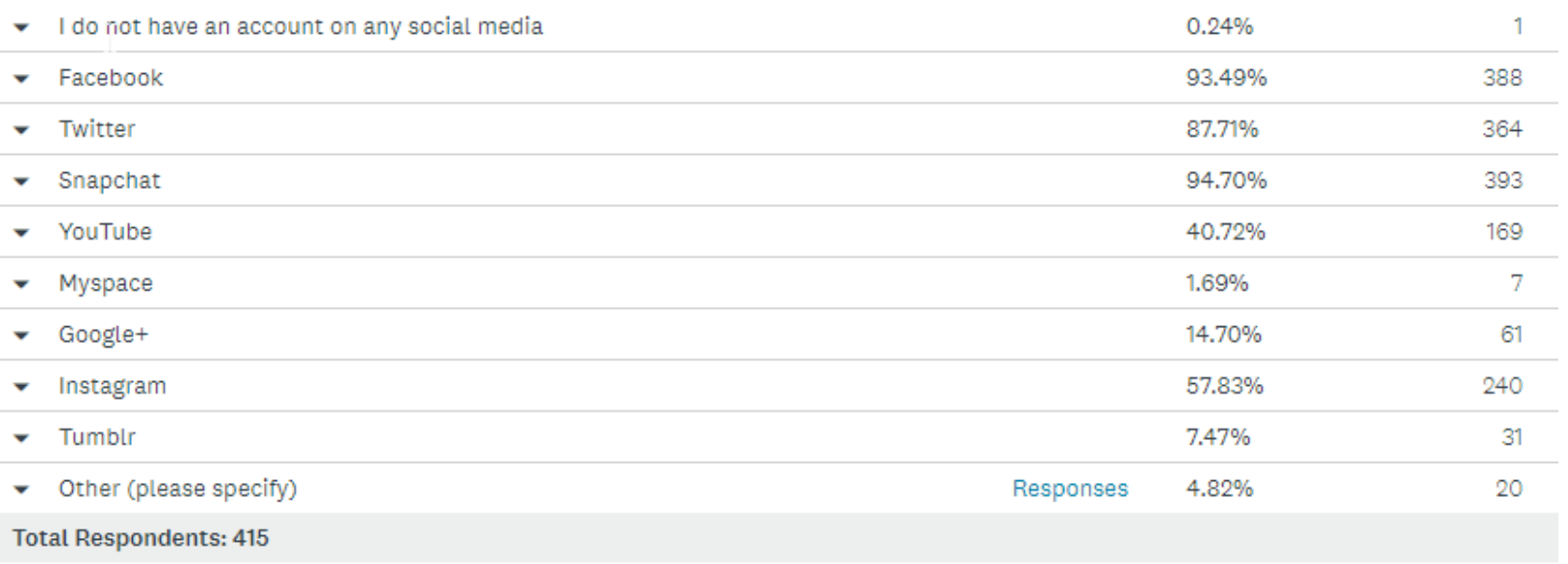

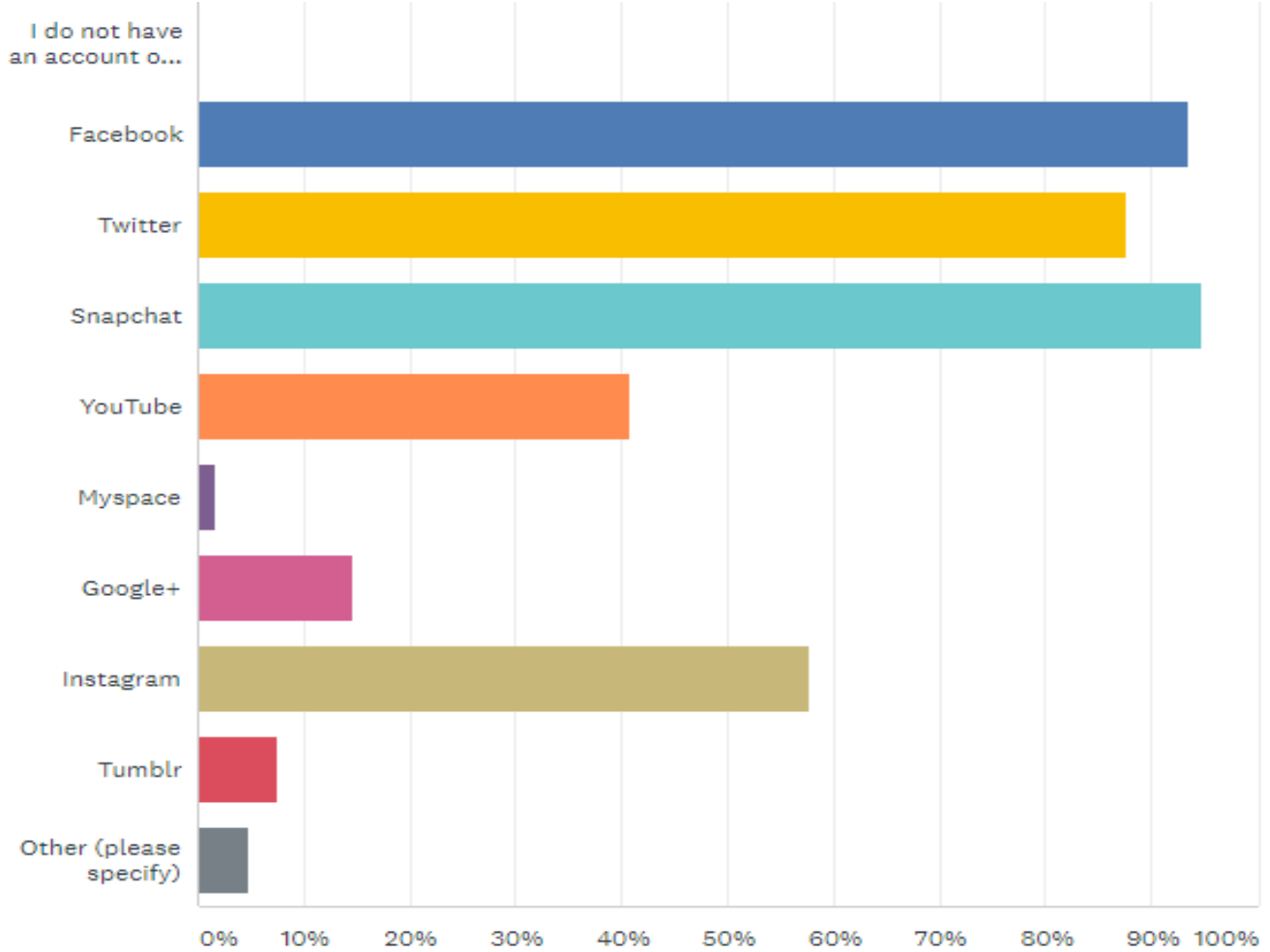

Figure 1. Most frequently used social media. 
In terms of frequency of usage, $52 \%$ of the respondents indicated that they accessed various social media several times a day, while $18 \%$ admitted accessing social media every hour of the day (Table 2).

Table 2

\section{Frequency of Social Media Usage}

\begin{tabular}{|c|c|c|}
\hline How often social media are used & Freque & \\
\hline Never & $0.96 \%$ & 4 \\
\hline A few times a month & $0.48 \%$ & 2 \\
\hline A few times a week & $4.34 \%$ & 18 \\
\hline About once a day & $24.82 \%$ & 103 \\
\hline Several times a day & $51.57 \%$ & 214 \\
\hline Every hour of the day & $17.83 \%$ & 74 \\
\hline TOTAL & & 415 \\
\hline
\end{tabular}

As shown in Table 3, in terms of gender variation, significant difference was found for the response option “about once a day” whereby more male students (33\%) responded in affirmative than female students (19\%). Conversely, for the response option "every hour of the day" more female students (25\%) responded in affirmative than male students (9\%).

Table 3

Frequency of Social Media Usage by Gender

\begin{tabular}{|c|c|c|c|c|c|c|c|c|}
\hline \multirow{2}{*}{\multicolumn{2}{|c|}{ Gender }} & \multicolumn{7}{|c|}{ Frequency and percent of respondents } \\
\hline & & NEVER $\boldsymbol{V}$ & $\begin{array}{l}\text { A FEW } \\
\text { TIMES A } \\
\text { MONTH }\end{array}$ & $\begin{array}{l}\text { A FEW } \\
\text { TIMES A } \\
\text { WEEK }\end{array}$ & $\begin{array}{l}\text { ABOUT } \\
\text { ONCEA } \\
\text { DAY }\end{array}$ & $\begin{array}{l}\text { SEVERAL } \\
\text { TIMES A } \\
\text { DAY }\end{array}$ & $\begin{array}{l}\text { EVERY HOUR } \\
\text { OF THE DAY }\end{array}$ & TOTAL \\
\hline & $\begin{array}{l}\text { Q90: Female } \\
\text { (A) }\end{array}$ & $\begin{array}{r}0.85 \% \\
2\end{array}$ & $\begin{array}{r}0.85 \% \\
2\end{array}$ & $\begin{array}{r}2.99 \% \\
7\end{array}$ & $\begin{array}{r}18.80 \% \\
44 \\
B\end{array}$ & $\begin{array}{r}51.71 \% \\
121\end{array}$ & $\begin{array}{r}24.79 \% \\
58 \\
\mathrm{~B}\end{array}$ & $\begin{array}{r}56.52 \% \\
234\end{array}$ \\
\hline$\nabla$ & $\begin{array}{l}\text { Q90: Male } \\
\text { (B) }\end{array}$ & $\begin{array}{r}1.11 \% \\
2\end{array}$ & $\begin{array}{r}0.00 \% \\
0\end{array}$ & $\begin{array}{r}6.11 \% \\
11\end{array}$ & $\begin{array}{r}32.78 \% \\
59 \\
\mathrm{~A}\end{array}$ & $\begin{array}{r}51.11 \% \\
92\end{array}$ & $\begin{array}{r}8.89 \% \\
16 \\
\mathrm{~A}\end{array}$ & $\begin{array}{r}43.48 \% \\
180\end{array}$ \\
\hline- & $\begin{array}{l}\text { Total } \\
\text { Respondents }\end{array}$ & 4 & 2 & 18 & 103 & 213 & 74 & 474 \\
\hline
\end{tabular}

Note. $* p=0.05,95 \%$ confidence.

In terms of age variation, the data showed significant differences for most of the dimensions. As shown in Table 4, the younger students accessed the social media more often than the older students.

With regard to the amount of time devoted to social media, approximately $32 \%$ of the students admitted using social media for 7-10 hours per day, 30\% spent 5-6 hours per day, and 29\% spent 1-2 hours a day (Table 5).

In terms of gender variation, the results showed significant difference for two dimensions. More females (14\%) used social media for 11 hours or more per day than males $(0.56 \%)$. Conversely, more male students used (37\%) social media for 1-2 hours per day than female (24\%) students (Table 6).

In terms of age variation, the data showed significant difference for two dimensions. As shown in Table 7, the younger students spent more time on social media than the older students. 
Table 4

Frequency of Social Media Usage by Age

Age groups

Frequency and percent of respondents

\begin{tabular}{|c|c|c|c|c|c|c|c|}
\hline$\checkmark$ & NEVER $~-$ & $\begin{array}{l}\text { A FEW } \\
\text { TIMES A } \\
\text { MONTH }\end{array}$ & $\begin{array}{l}\text { A FEW } \\
\text { TIMES A } \\
\text { WEEK }\end{array}$ & $\begin{array}{l}\text { ABOUT } \\
\text { ONCE A } \\
\text { DAY }\end{array}$ & $\begin{array}{l}\text { SEVERAL } \\
\text { TIMES A } \\
\text { DAY }\end{array}$ & $\begin{array}{l}\text { EVERY HOUR } \\
\text { OF THE DAY }\end{array}$ & TOTAL \\
\hline $\begin{array}{l}\text { Q89: } 18-20 \\
\text { (A) }\end{array}$ & $\begin{array}{r}0.76 \% \\
2\end{array}$ & $\begin{array}{r}0.38 \% \\
1\end{array}$ & $\begin{array}{r}1.91 \% \\
5 \\
\mathrm{C}\end{array}$ & $\begin{array}{r}14.89 \% \\
39 \\
B C\end{array}$ & $\begin{array}{r}57.63 \% \\
151 \\
\mathrm{C}\end{array}$ & $\begin{array}{r}24.43 \% \\
64 \\
\mathrm{BC}\end{array}$ & $\begin{array}{r}63.29 \% \\
262\end{array}$ \\
\hline $\begin{array}{l}\text { Q89: } 21-25 \\
\text { (B) }\end{array}$ & $\begin{array}{r}1.82 \% \\
2\end{array}$ & $\begin{array}{r}0.91 \% \\
1\end{array}$ & $\begin{array}{r}2.73 \% \\
3 \\
\mathrm{C}\end{array}$ & $\begin{array}{r}29.09 \% \\
32 \\
\text { AC }\end{array}$ & $\begin{array}{r}57.27 \% \\
63 \\
\mathrm{C}\end{array}$ & $\begin{array}{r}8.18 \% \\
9 \\
\mathrm{~A}\end{array}$ & $\begin{array}{r}26.57 \% \\
110\end{array}$ \\
\hline $\begin{array}{l}\text { Q89: } 26 \text { and } \\
\text { above (C) }\end{array}$ & $\begin{array}{r}0.00 \% \\
0\end{array}$ & $\begin{array}{r}0.00 \% \\
0\end{array}$ & $\begin{array}{r}23.81 \% \\
10 \\
\mathrm{AB}\end{array}$ & $\begin{array}{r}76.19 \% \\
32 \\
\mathrm{AB}\end{array}$ & $\begin{array}{r}0.00 \% \\
0 \\
A B\end{array}$ & $\begin{array}{r}0.00 \% \\
0 \\
\mathrm{~A}\end{array}$ & $\begin{array}{r}10.14 \% \\
42\end{array}$ \\
\hline $\begin{array}{l}\text { Total } \\
\text { Respondents }\end{array}$ & 4 & 2 & 18 & 103 & 214 & 73 & 414 \\
\hline
\end{tabular}

Note. ${ }^{*} p=0.05,95 \%$ confidence.

Table 5

Amount of Time Spent on Social Media

Amount of time

Frequency and percent of respondents

\begin{tabular}{llr} 
& & 5 \\
\hline $1-2$ hours & $1.20 \%$ & 122 \\
\hline $5-6$ hours & $29.40 \%$ & 124 \\
\hline $7-10$ hours & $29.88 \%$ & 131 \\
\hline 11 hours or more & $31.57 \%$ & 33 \\
\hline \multicolumn{1}{l}{ TOTAL } & $7.95 \%$ & 415
\end{tabular}

Table 6

Amount of Time Spent on Social Media by Gender

Gender

Frequency and percent of respondents

\begin{tabular}{|c|c|c|c|c|c|c|}
\hline 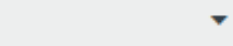 & 0 HOURS & 1-2 HOURS & 5-6 HOURS & 7-10 HOURS & 11 HOURS OR MORE & TOTAL \\
\hline $\begin{array}{l}\text { Q90: Female } \\
\text { (A) }\end{array}$ & $\begin{array}{r}0.43 \% \\
1\end{array}$ & $\begin{array}{r}23.93 \% \\
56 \\
\mathrm{~B}\end{array}$ & $\begin{array}{r}31.62 \% \\
74\end{array}$ & $\begin{array}{r}30.34 \% \\
71\end{array}$ & $\begin{array}{r}13.68 \% \\
32 \\
\mathrm{~B}\end{array}$ & $\begin{array}{r}56.52 \% \\
234\end{array}$ \\
\hline $\begin{array}{l}\text { Q90: Male } \\
\text { (B) }\end{array}$ & $\begin{array}{r}2.22 \% \\
4\end{array}$ & $\begin{array}{r}36.67 \% \\
66 \\
\mathrm{~A}\end{array}$ & $\begin{array}{r}27.78 \% \\
50\end{array}$ & $\begin{array}{r}32.78 \% \\
59\end{array}$ & $\begin{array}{r}0.56 \% \\
1 \\
\mathrm{~A}\end{array}$ & $\begin{array}{r}43.48 \% \\
180\end{array}$ \\
\hline $\begin{array}{l}\text { Total } \\
\text { Respondents }\end{array}$ & 5 & 122 & 724 & 130 & 33 & 474 \\
\hline
\end{tabular}

Note. ${ }^{*} p=0.05,95 \%$ confidence. 
Table 7

Amount of Time Spent on Social Media by Age

Age groups Frequency and percent of respondents

\begin{tabular}{|c|c|c|c|c|c|c|c|}
\hline & $\nabla$ & 0 HOURS $\quad$ & 1-2 HOURS & 5-6 HOURS & 7-10 HOURS & 11 HOURS OR MORE & TOTAL \\
\hline$\nabla$ & $\begin{array}{l}\text { Q89: } 18-20 \\
\text { (A) }\end{array}$ & $\begin{array}{r}0.76 \% \\
2\end{array}$ & $\begin{array}{r}14.89 \% \\
39 \\
B C\end{array}$ & $\begin{array}{r}39.31 \% \\
103\end{array}$ & $\begin{array}{r}32.82 \% \\
86\end{array}$ & $\begin{array}{r}12.21 \% \\
32 \\
B C\end{array}$ & $\begin{array}{r}63.29 \% \\
262\end{array}$ \\
\hline 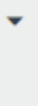 & $\begin{array}{l}\text { Q89: } 21-25 \\
\text { (B) }\end{array}$ & $\begin{array}{r}2.73 \% \\
3\end{array}$ & $\begin{array}{r}37.27 \% \\
41 \\
\mathrm{AC}\end{array}$ & $\begin{array}{r}19.09 \% \\
21\end{array}$ & $\begin{array}{r}40.00 \% \\
44\end{array}$ & $\begin{array}{r}0.91 \% \\
1 \\
\mathrm{~A}\end{array}$ & $\begin{array}{r}26.57 \% \\
110\end{array}$ \\
\hline$\nabla$ & $\begin{array}{l}\text { Q89: } 26 \text { and } \\
\text { above }(C)\end{array}$ & $\begin{array}{r}0.00 \% \\
0\end{array}$ & $\begin{array}{r}100.00 \% \\
42 \\
A B\end{array}$ & $\begin{array}{r}0.00 \% \\
0\end{array}$ & $\begin{array}{r}0.00 \% \\
0\end{array}$ & $\begin{array}{r}0.00 \% \\
0 \\
\mathrm{~A}\end{array}$ & $\begin{array}{r}10.14 \% \\
42\end{array}$ \\
\hline$\nabla$ & $\begin{array}{l}\text { Total } \\
\text { Respondents }\end{array}$ & 5 & 122 & 124 & 130 & 33 & 414 \\
\hline
\end{tabular}

Note. $* p=0.05,95 \%$ confidence.

\section{How Does Generation Z Consume News?}

The results revealed that $92 \%$ of Gen Zers used their smartphones to check the news online, and most students used multiple sources of news (Table 8). As shown in Table 9, there was no significant gender difference for most of the dimensions of this variable. However, in terms of age difference, data showed that older students preferred to get their news from television and younger students chose to use their laptops to access the news online (Table 10).

Table 8

Devices Used to Access the News

Types of devices

Frequency and percent of respondents

\begin{tabular}{llr}
\hline I do not follow news & $3.38 \%$ & 14 \\
\hline Smartphone & $91.79 \%$ & 380 \\
\hline Laptop & $46.14 \%$ & 197 \\
\hline iPad & $6.04 \%$ & 25 \\
\hline TV & $48.31 \%$ & 200 \\
\hline Radio & $6.52 \%$ & 27 \\
\hline Hard copy newspapers & $4.83 \%$ & 20 \\
\hline Total Respondents: 414 & $2.66 \%$ & 17 \\
\hline
\end{tabular}


Table 9

Devices Used to Access the News by Gender

Gender

Frequency and percent of respondents

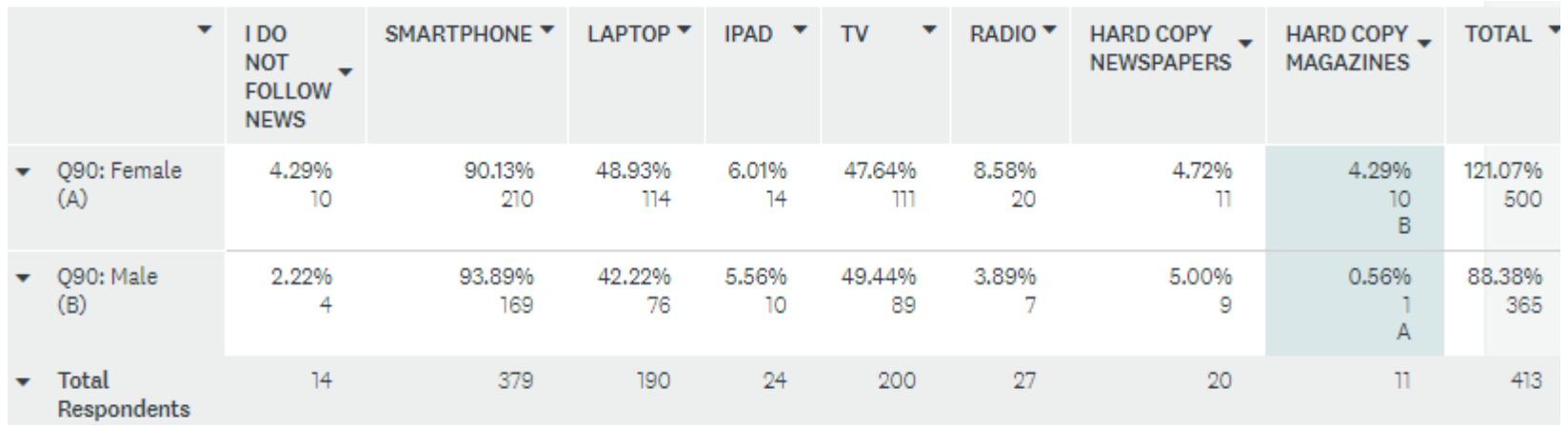

Note. $* p=0.05,95 \%$ confidence.

Table 10

Devices Used to Access the News by Age

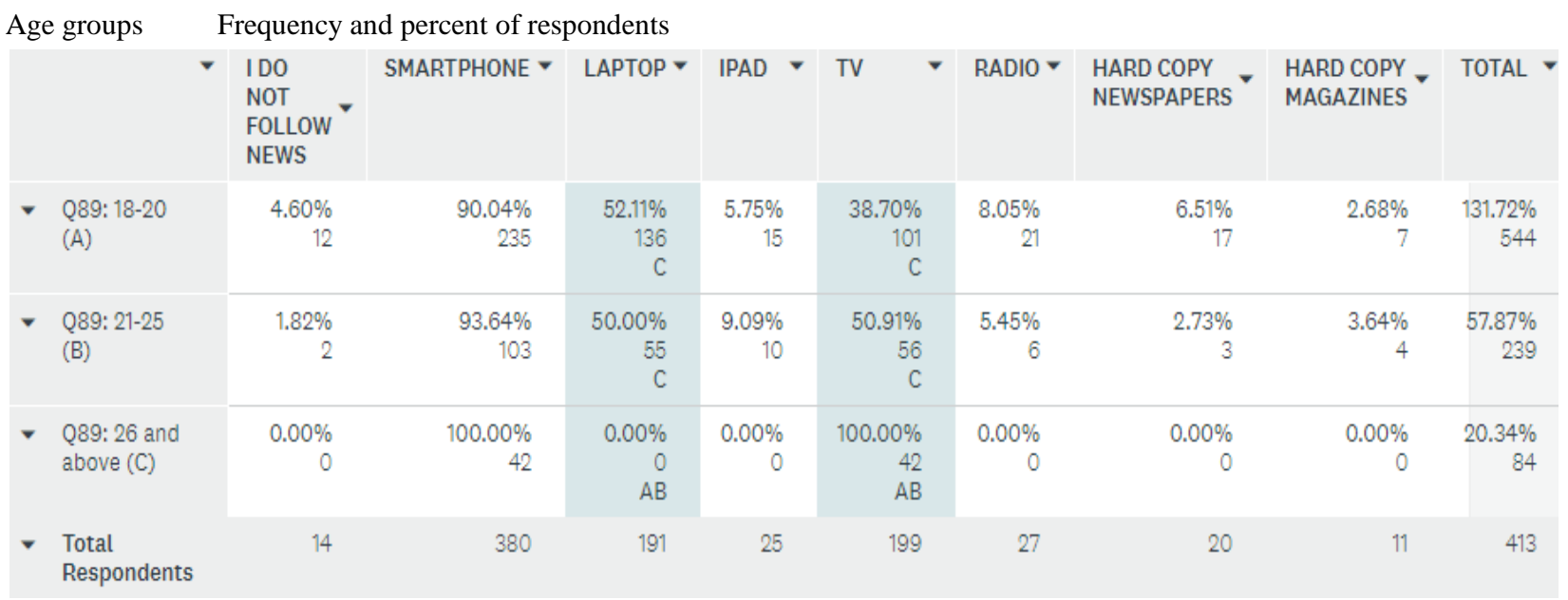

Note. $* p=0.05,95 \%$ confidence.

With regard to sources of news, about $79 \%$ of the students obtained their news from online newspapers and magazines, while 58\% also watched television news (Table 11). Furthermore, $60 \%$ of them indicated that they also got their news on Facebook (50\%) and Twitter (50\%). Only about $8 \%$ stated that they read hardcopy newspapers and magazines, and $7 \%$ also got their news from radio (Table 11). About 3\% of the students admitted that they did not follow the news at all. As shown in the table below, there was no significant gender difference for this variable (Table 12). However, in terms of age variation, data showed that larger percentage of younger students used Facebook and Twitter to get their news compared to older students who preferred television news (Table 13). 
Table 11

Sources of News Used by Generation Z

Sources of news

Frequency and percent of respondents

\begin{tabular}{|c|c|c|}
\hline - I do not follow news & $3.38 \%$ & 14 \\
\hline Online newspapers and magazines & $78.50 \%$ & 325 \\
\hline Hardcopy newspapers and magazines & $8.45 \%$ & 35 \\
\hline - Radio & $7.25 \%$ & 30 \\
\hline - Television & $57.73 \%$ & 239 \\
\hline Twitter & $50.48 \%$ & 209 \\
\hline - Facebook & $60.14 \%$ & 249 \\
\hline
\end{tabular}

Table 12

Sources of News Used by Gender

\begin{tabular}{|c|c|c|c|c|c|c|c|c|}
\hline \multirow[t]{2}{*}{ Gender } & \multicolumn{8}{|c|}{ Frequency and percent of respondents } \\
\hline & $\begin{array}{l}\text { I DO } \\
\text { NOT } \\
\text { FOLLOW } \\
\text { NEWS }\end{array}$ & $\begin{array}{l}\text { ONLINE } \\
\text { NEWSPAPERS } \\
\text { AND } \\
\text { MAGAZINES }\end{array}$ & $\begin{array}{l}\text { HARDCOPY } \\
\text { NEWSPAPERS } \\
\text { AND } \\
\text { MAGAZINES }\end{array}$ & RADIO - & TELEVISION & TWITTER $~-$ & FACEBOOK $>$ & TOTAL $\vee$ \\
\hline $\begin{array}{l}\text { Q90: Female } \\
\text { (A) }\end{array}$ & $\begin{array}{r}1.51 \% \\
10\end{array}$ & $\begin{array}{r}29.35 \% \\
194\end{array}$ & $\begin{array}{r}3.63 \% \\
24\end{array}$ & $\begin{array}{r}3.48 \% \\
23\end{array}$ & $\begin{array}{r}20.27 \% \\
134\end{array}$ & $\begin{array}{r}19.52 \% \\
729\end{array}$ & $\begin{array}{r}22.24 \% \\
147\end{array}$ & $\begin{array}{r}160.05 \% \\
661\end{array}$ \\
\hline $\begin{array}{l}\text { Q90: Male } \\
\text { (B) }\end{array}$ & $\begin{array}{r}0.91 \% \\
4\end{array}$ & $\begin{array}{r}29.45 \% \\
729\end{array}$ & $\begin{array}{r}2.51 \% \\
11\end{array}$ & $\begin{array}{r}1.60 \% \\
7\end{array}$ & $\begin{array}{r}23.97 \% \\
105\end{array}$ & $\begin{array}{r}18.26 \% \\
80\end{array}$ & $\begin{array}{r}23.29 \% \\
102\end{array}$ & $\begin{array}{r}106.05 \% \\
438\end{array}$ \\
\hline $\begin{array}{l}\text { Total } \\
\text { Respondents }\end{array}$ & 14 & 323 & 35 & 30 & 239 & 209 & 249 & 413 \\
\hline
\end{tabular}

Table 13

Sources of News Used by Age

\begin{tabular}{|c|c|c|c|c|c|c|c|c|c|}
\hline \multirow{2}{*}{\multicolumn{2}{|c|}{ Age groups }} & \multicolumn{3}{|c|}{ Frequency and percent of respondents } & \multirow[b]{2}{*}{ RADIO V } & \multirow[b]{2}{*}{ TELEVISION } & \multirow[b]{2}{*}{ TWITTER $~-$} & \multirow[b]{2}{*}{ FACEBOOK - } & \multirow[b]{2}{*}{ TOTAL $\vee$} \\
\hline & & $\begin{array}{l}\text { IDO } \\
\text { NOT } \\
\text { FOLLOW } \\
\text { NEWS }\end{array}$ & $\begin{array}{l}\text { ONLINE } \\
\text { NEWSPAPERS } \\
\text { AND } \\
\text { MAGAZINES }\end{array}$ & $\begin{array}{l}\text { HARDCOPY } \\
\text { NEWSPAPERS } \\
\text { AND } \\
\text { MAGAZINES }\end{array}$ & & & & & \\
\hline 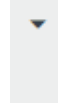 & $\begin{array}{l}\text { Q89: } 18-20 \\
\text { (A) }\end{array}$ & $\begin{array}{r}1.77 \% \\
13\end{array}$ & $\begin{array}{r}27.56 \% \\
202\end{array}$ & $\begin{array}{r}3.55 \% \\
26\end{array}$ & $\begin{array}{r}3.27 \% \\
24\end{array}$ & $\begin{array}{r}16.64 \% \\
122 \\
\text { BC }\end{array}$ & $\begin{array}{r}22.37 \% \\
164 \\
\mathrm{BC}\end{array}$ & $\begin{array}{r}24.83 \% \\
182 \\
\mathrm{C}\end{array}$ & $\begin{array}{r}177.48 \% \\
733\end{array}$ \\
\hline$v$ & $\begin{array}{l}\text { Q89: } 21-25 \\
\text { (B) }\end{array}$ & $\begin{array}{r}0.35 \% \\
1\end{array}$ & $\begin{array}{r}28.62 \% \\
81\end{array}$ & $\begin{array}{r}2.83 \% \\
8\end{array}$ & $\begin{array}{r}2.12 \% \\
6\end{array}$ & $\begin{array}{r}26.50 \% \\
75 \\
\mathrm{AC}\end{array}$ & $\begin{array}{r}15.90 \% \\
45 \\
\mathrm{AC}\end{array}$ & $\begin{array}{r}23.67 \% \\
67 \\
\mathrm{C}\end{array}$ & $\begin{array}{r}68.52 \% \\
283\end{array}$ \\
\hline$v$ & $\begin{array}{l}\text { Q89: } 26 \text { and } \\
\text { above (C) }\end{array}$ & $\begin{array}{r}0.00 \% \\
0\end{array}$ & $\begin{array}{r}50.00 \% \\
42\end{array}$ & $\begin{array}{r}0.00 \% \\
0\end{array}$ & $\begin{array}{r}0.00 \% \\
0\end{array}$ & $\begin{array}{r}50.00 \% \\
42 \\
\mathrm{AB}\end{array}$ & $\begin{array}{r}0.00 \% \\
0 \\
\mathrm{AB}\end{array}$ & $\begin{array}{r}0.00 \% \\
0 \\
A B\end{array}$ & $\begin{array}{r}20.34 \% \\
84\end{array}$ \\
\hline 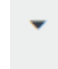 & $\begin{array}{l}\text { Total } \\
\text { Respondents }\end{array}$ & 14 & 325 & 34 & 30 & 239 & 209 & 249 & 413 \\
\hline
\end{tabular}

Note. $* p=0.05,95 \%$ confidence. 
With regard to the type of news they consume, about $80 \%$ indicated that they paid attention to local news, 89\% paid attention to national news, and 77\% followed international news. Moreover, about $43 \%$ consumed sports news, $39 \%$ paid attention to science news, and $44 \%$ followed celebrity news (Table 14). In terms of gender variation, the results showed significant difference for several dimensions (Table 15). More male students consumed (58\%) sports news compared to female students (32\%), and more female students followed celebrity news (55\%) compared to male students (30\%). The results were at $95 \%$ confidence level, $\mathrm{p}=0.05$.

Table 14

Types of News Consumed by Generation Z

Types of news

Frequency and percent of respondents

\begin{tabular}{lll} 
- I do not follow news & $3.38 \%$ & 14 \\
\hline - Local & $80.43 \%$ & 333 \\
\hline National & $89.13 \%$ & 369 \\
\hline - International & $77.05 \%$ & 319 \\
\hline - Sports & $43.24 \%$ & 179 \\
\hline - Science & $38.65 \%$ & 760 \\
\hline Celebrity & $44.20 \%$ & 183
\end{tabular}

Total Respondents: 414

Table 15

Sources of News Consumed by Gender

Gender

Frequency and percent of respondents

\begin{tabular}{|c|c|c|c|c|c|c|c|c|}
\hline$\nabla$ & $\begin{array}{l}\text { I DO } \\
\text { NOT } \\
\text { FOLLOW } \\
\text { NEWS }\end{array}$ & LOCAL $\mathbf{}$ & NATIONAL - & INTERNATIONAL - & SPORTS - & SCIENCE - & CELEBRITY - & TOTAL \\
\hline $\begin{array}{l}\text { Q90: Female } \\
\text { (A) }\end{array}$ & $\begin{array}{r}5.15 \% \\
12 \\
\mathrm{~B}\end{array}$ & $\begin{array}{r}81.12 \% \\
189\end{array}$ & $\begin{array}{r}88.41 \% \\
206\end{array}$ & $\begin{array}{r}76.82 \% \\
179\end{array}$ & $\begin{array}{r}32.19 \% \\
75 \\
\mathrm{~B}\end{array}$ & $\begin{array}{r}36.91 \% \\
86\end{array}$ & $\begin{array}{r}55.36 \% \\
729 \\
\mathrm{~B}\end{array}$ & $\begin{array}{r}212.11 \% \\
876\end{array}$ \\
\hline $\begin{array}{l}\text { Q90: Male } \\
\text { (B) }\end{array}$ & $\begin{array}{r}1.11 \% \\
2 \\
\mathrm{~A}\end{array}$ & $\begin{array}{r}79.44 \% \\
743\end{array}$ & $\begin{array}{r}90.00 \% \\
162\end{array}$ & $\begin{array}{r}77.22 \% \\
139\end{array}$ & $\begin{array}{r}57.78 \% \\
104 \\
\mathrm{~A}\end{array}$ & $\begin{array}{r}40.56 \% \\
73\end{array}$ & $\begin{array}{r}30.00 \% \\
54 \\
\mathrm{~A}\end{array}$ & $\begin{array}{r}163.92 \% \\
677\end{array}$ \\
\hline $\begin{array}{l}\text { Total } \\
\text { Respondents }\end{array}$ & 14 & 332 & 368 & 318 & 179 & 159 & 183 & 413 \\
\hline
\end{tabular}

Note. ${ }^{*} p=0.05,95 \%$ confidence.

In terms of age variation, data showed that larger percentage of younger students consumed sports news and celebrity news compared to older students. Conversely, larger percentage of older students consumed science news compared to younger students (Table 16). 
Table 16

Sources of News Consumed by Age

\begin{tabular}{|c|c|c|c|c|c|c|c|c|c|}
\hline \multicolumn{2}{|c|}{ Age groups } & \multicolumn{3}{|c|}{ Frequency and percent of respondents } & \multirow[b]{2}{*}{ INTERNATIONAL • } & \multirow[b]{2}{*}{ SPORTS V } & \multirow[b]{2}{*}{ SCIENCE $>$} & \multirow[b]{2}{*}{ CELEBRITY $\boldsymbol{V}$} & \multirow[b]{2}{*}{ TOTAL } \\
\hline & 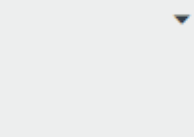 & $\begin{array}{l}\text { I DO } \\
\text { NOT } \\
\text { FOLLOW } \\
\text { NEWS }\end{array}$ & LOCAL $>$ & NATIONAL & & & & & \\
\hline$v$ & $\begin{array}{l}\text { Q89: } 18-20 \\
\text { (A) }\end{array}$ & $\begin{array}{r}5.36 \% \\
14 \\
\mathrm{~B}\end{array}$ & $\begin{array}{r}75.86 \% \\
198\end{array}$ & $\begin{array}{r}85.82 \% \\
224\end{array}$ & $\begin{array}{r}72.41 \% \\
189\end{array}$ & $\begin{array}{r}45.21 \% \\
118 \\
\mathrm{C}\end{array}$ & $\begin{array}{r}29.12 \% \\
76 \\
\text { BC }\end{array}$ & $\begin{array}{r}52.11 \% \\
136 \\
\mathrm{C}\end{array}$ & $\begin{array}{r}231.23 \% \\
955\end{array}$ \\
\hline 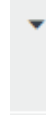 & $\begin{array}{l}\text { Q89: } 21-25 \\
\text { (B) }\end{array}$ & $\begin{array}{r}0.00 \% \\
0 \\
\mathrm{~A}\end{array}$ & $\begin{array}{r}84.55 \% \\
93\end{array}$ & $\begin{array}{r}93.64 \% \\
103\end{array}$ & $\begin{array}{r}79.09 \% \\
87\end{array}$ & $\begin{array}{r}46.36 \% \\
51\end{array}$ & $\begin{array}{r}53.64 \% \\
59 \\
\mathrm{~A}\end{array}$ & $\begin{array}{r}42.73 \% \\
47 \\
\mathrm{C}\end{array}$ & $\begin{array}{r}106.54 \% \\
440\end{array}$ \\
\hline 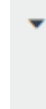 & $\begin{array}{l}\text { Q89: } 26 \text { and } \\
\text { above }(C)\end{array}$ & $\begin{array}{r}0.00 \% \\
0\end{array}$ & $\begin{array}{r}100.00 \% \\
42\end{array}$ & $\begin{array}{r}100.00 \% \\
42\end{array}$ & $\begin{array}{r}100.00 \% \\
42\end{array}$ & $\begin{array}{r}23.81 \% \\
10 \\
\mathrm{~A}\end{array}$ & $\begin{array}{r}59.52 \% \\
25 \\
\mathrm{~A}\end{array}$ & $\begin{array}{r}0.00 \% \\
0 \\
A B\end{array}$ & $\begin{array}{r}38.98 \% \\
161\end{array}$ \\
\hline$v$ & $\begin{array}{l}\text { Total } \\
\text { Respondents }\end{array}$ & 14 & 333 & 369 & 318 & 179 & 160 & 183 & 413 \\
\hline
\end{tabular}

Note. $* p=0.05,95 \%$ confidence.

With regard to the amount of time devoted to consuming news, $82 \%$ of the respondents indicated that they spent 1-2 hours in a typical day for news consumption while $11 \%$ did not spend any time consuming news (Table 17). As shown in the table below, there was no significant gender difference for this variable (Table 18). In terms of age variation, older students were more likely to devote more time in news consumption on a daily basis (Table 19).

Table 17

Amount of Time Devoted to Consuming News

Amount of time

Frequency and percent of respondents

\begin{tabular}{llr}
\hline O hours & $11.33 \%$ & 47 \\
\hline $1-2$ & $82.41 \%$ & 342 \\
\hline $3-4$ & $4.82 \%$ & 20 \\
\hline $5-6$ & $1.20 \%$ & 5 \\
\hline 7 hours or more & $0.24 \%$ & 1 \\
\hline TOTAL & & 415
\end{tabular}

Table 18

Amount of Time Devoted to Consuming News by Gender

Amount of time Frequency and percent of respondents

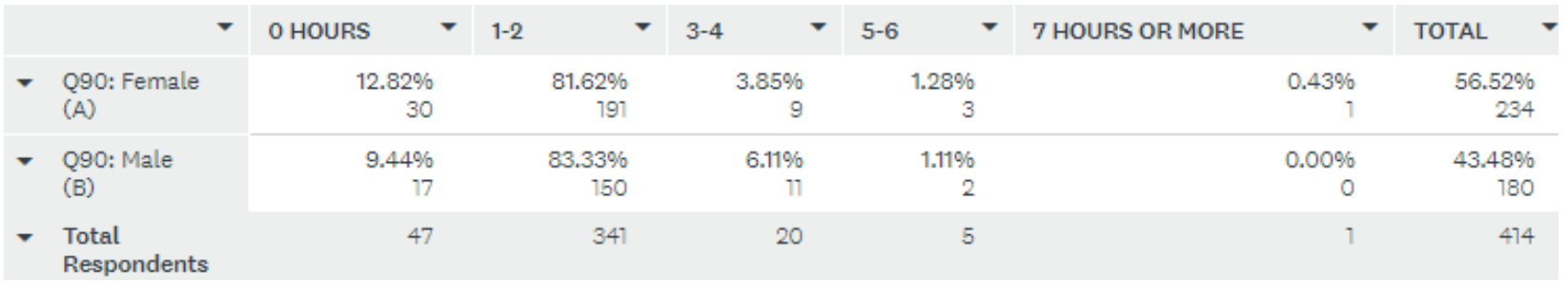


Table 19

Amount of Time Devoted to Consuming News by Age

Age groups

Frequency and percent of respondents

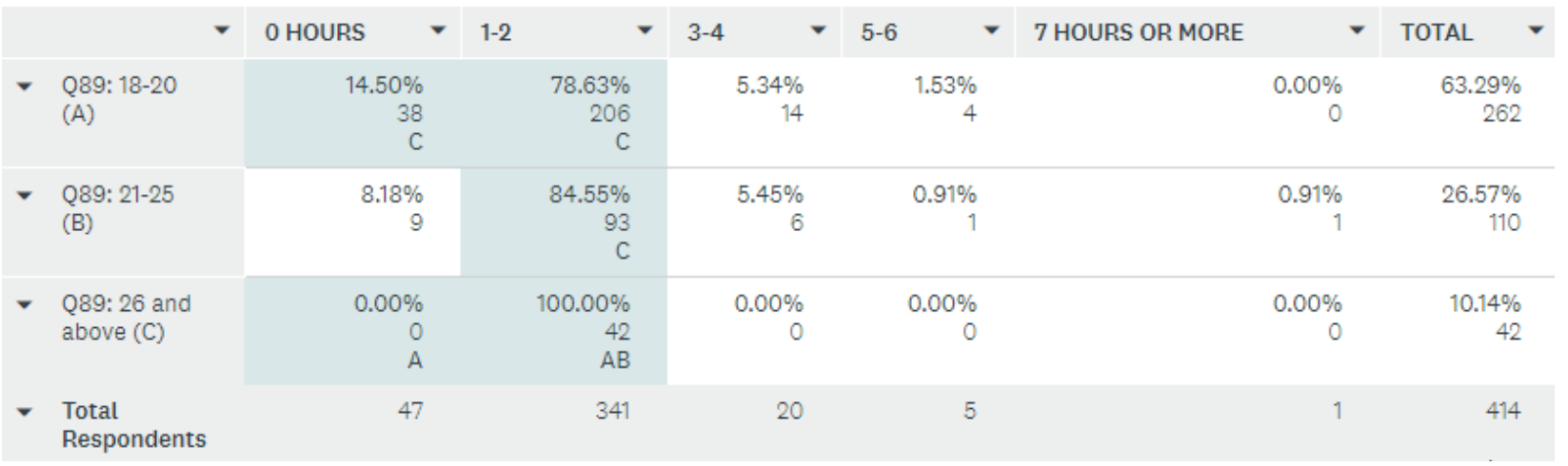

Note. $* p=0.05,95 \%$ confidence.

In terms of exposure to fake news, about 93\% of the students indicated that they had seen some news on social media that turned out to be fake news (Table 20). As shown in Tables 21-22, there was no significant gender or age difference for this variable.

Table 20

Exposure to Fake News on Social Media

Responses

Frequency and percent of respondents

\begin{tabular}{lrr} 
Yes & $92.51 \%$ & 383 \\
\hline No & $1.69 \%$ & 7 \\
\hline Not sure & $5.31 \%$ & 22 \\
\hline I do not use social media & $0.48 \%$ & 2 \\
TOTAL & & 414 \\
\end{tabular}

Table 21

Exposure to Fake News on Social Media by Gender

Gender Frequency and percent of respondents

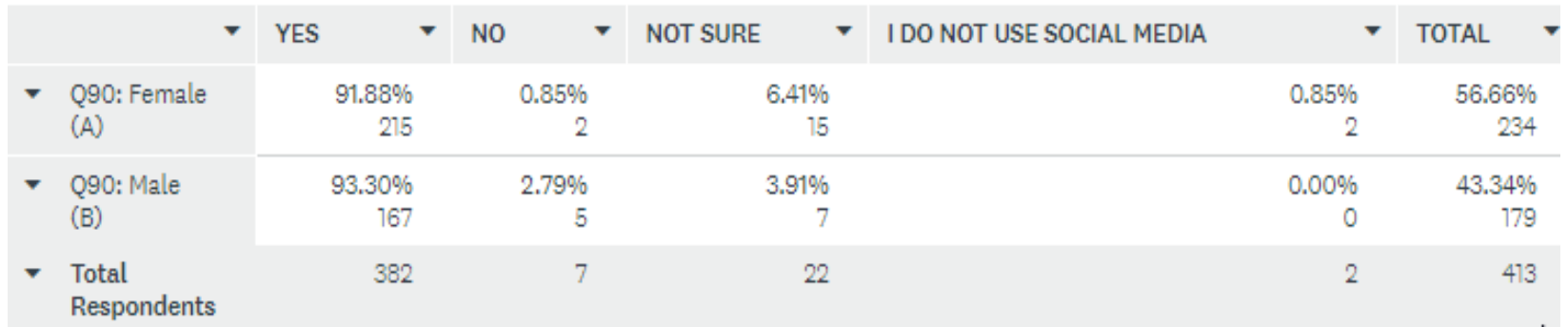


Table 22

Exposure to Fake News on Social Media by Age

\begin{tabular}{l|r|r|r|r|r|}
\hline Age groups & \multicolumn{2}{c}{ Frequency and percent of respondents } & & \\
\hline
\end{tabular}

Note. $* p=0.05,95 \%$ confidence.

\section{Discussion and Conclusions}

This study examined post-millennials' news consumption habits and perception of fake news in the social media. A survey was completed by a non-random sample of 415 students at State University of New York in Oneonta during the academic year 2017-2018. The most significant findings are discussed and interpreted below.

The results revealed that almost all Gen Zers (99\%) own a smartphone, and 98\% of them use their smartphones to connect to the Internet most of the time. More than half of Gen Zers spend nine hours or more in a typical day using their smartphones, and nearly half of them spend 3-8 hours per day on their smartphones. Data did not show any significant difference in terms of gender or age groups, which indicates that both males and females, and younger as well as older students are spending a significant amount of time on their smartphones on a daily basis.

With regard to the use of social media, the results revealed that Snapchat, Instagram, Facebook, and Twitter are the most widely used social media among Generation $\mathrm{Z}$ with majority of them having multiple social media accounts. In terms of frequency of usage, more than half of Gen Zers indicated that they accessed various social media several times a day, while nearly one in five admitted accessing social media every hour of the day. In terms of gender variation, data showed that more female students accessed various social media every hour of the day compared to males. As for age variation, the findings revealed that the younger students accessed the social media more often than the older students.

With regard to the amount of time devoted to social media, nearly one-third of the students admitted using social media for 7-10 hours per day, and slightly less than one-third of the students spent 5-6 hours per day on social media. There were no significant differences in terms of gender for most of the dimensions. However, a greater percentage of younger students spent more time on social media than older students.

With regard to news consumption habits of Generation Z, data analysis revealed that nine in 10 students used their smartphones to check the news online, and most students used multiple sources of news. About four-fifths of the students obtained their news from online newspapers and magazines, while three-fifths of them also used social media for obtaining news. Although there was no significant gender difference, younger students were more likely to get their news from social media. As for the amount of time devoted to consuming 
news, four-fifths of the students indicated that they spent 1-2 hours in a typical day for news consumption. Older students were more likely to devote more time for news consumption on a daily basis compared to younger students. There was no significant gender difference for this variable. In terms of exposure to fake news, nine in 10 students indicated that they had seen some news on social media that turned out to be fake news. There was no significant gender or age difference for this variable.

These findings may have significant implications for social media as they plan to counter the proliferation of fake news on their platforms. This research was based on a non-random sample and focused on post-millennials, and thereby limiting the ability to generalize the results to the larger population. Future research with a random sample could focus on additional variables and dimensions relating to the usage of social media, and its implications for the personal, educational, and professional lives of all demographics.

\section{References}

Al-Menayes, J. (2016). The fear of missing out scale: Validation of the Arabic version and correlation with social media addiction. International Journal of Applied Psychology, 6, 41-46.

Andrew, H. (2018, June 5). Snapchat publishes new insights into Generation Z. Social Media Today. Retrieved from https://www.socialmediatoday.com/news/snapchat-publishes-new-insights-into-generation-z-infographic/524940/

Bian, M., \& Leung, L. (2014). Linking loneliness, shyness, smartphone addiction symptoms, and patterns of smartphone use to social capital. Social Science Computer Review, 33(1), 61-79.

Billieux, J., Maurage, P., Lopez-Fernandez, O., Kuss, D. J., \& Griffiths, M. D. (2015). Can disordered mobile phone use be considered a behavioral addiction? An update on current evidence and a comprehensive model for future research. Current Addiction Report, 2, 156-162.

Bragazzi, N. L., \& Del Puente, G. (2014). A proposal for including nomophobia in the new DSMV. Psychology Research and Behavior Management, 7, 155-160.

Buglass, S. L., Binder, J. F., Betts, L. R., \& Underwood, J. D. M. (2017). Motivators of online vulnerability: The impact of social network site use and FOMO. Computers in Human Behavior, 66, 248-255.

Caplan, S. E. (2003). Preference for online social interaction: A theory of problematic Internet use and psychosocial well-being. Communication Research, 30, 625-648.

Chen, Y., Liang, C., Mai, X., \& Zhong, Q. (2016). General deficit in inhibitory control of excessive smartphone users: Evidence from an event-related potential study. Frontiers in Psychology, 7, 511.

Christofides, E., Muise, A., \& Desmarais, S. (2012). Risky disclosures on Facebook: The effect of having a bad experience on online behavior. Journal of Adolescent Research, 27(6), 714-731.

Deloitte Insights. (2018). Deloitte digital media trends survey (12th ed.).

Hawi, N., \& Samaha, M. (2016). The relations among social media addiction, self-esteem, and life satisfaction in university students. Social Science Computer Review, 35(5), 576-586.

Hermida, A., Fletcher, F., Korell., D., \& Logan, D. (2012). Share, like, recommend: Decoding the social media news consumer. Journalism Studies, 13(5-6), 815-824.

Hong, F., \& Chiu, D. (2012). A model of the relationship between psychological characteristics, mobile phone addiction and use of mobile phones by Taiwanese university female students. Computer Human Behavior, 28, 2152-2159.

Jarvis, S., Jomini, N., Austin, S., \&. Gilliland, A. (2009). College students, news use, and trust. Communication Research Reports, 26(1), 30-39.

Jeong, S., Kim, H., Yum, J., \& Hwang, Y. (2016). What type of content are smartphone users addicted to?: SNS vs. games. Computers in Human Behavior, 54, 10-17.

King, D., Delfabbro, P., \& Griffiths, M. (2010). The convergence of gambling and digital media: Implications for gambling in young people. Journal of Gambling Studies, 26, 175-187.

Kross, E., Verduyn, P., Demiralp, E., Park, J., Lee, D. S., \& Lin, N. (2013). Facebook use predicts declines in subjective well-being in young adults. PLOS One, 8(8), e69841.

Kuss, D. J., \& Griffiths, M. D. (2011). Online social networking and addiction-a review of the psychological literature. International Journal of Environmental Research. Public Health, 8, 3528-3552. 
Kuss, D. J., \& Griffiths, M. D. (2012). Internet gaming addiction: A systematic review of empirical research. International Journal of Mental Health Addiction, 10, 278-296.

Lee, J., Sung, M., Song, S., Lee, Y., Lee, J., Cho, S., Park, M., \& Shin, Y. (2016). Psychological factors associated with smartphone addiction in South Korean adolescents. The Journal of Early Adolescence, 38(3), 288-302.

Lin, Y. C., Lee, P. H., Lin, S. H., Lin, L. R., \& Chang, T. B. (2015). Time distortion associated with smartphone addiction: Identifying smartphone addiction via a mobile application. Journal of Psychiatric Research, 65, 139-145.

Lopez-Fernandez, O., Kuss, D. J., Griffiths, M. D., \& Billieux, J. (2015). The conceptualization and assessment of problematic mobile phone use. In Encyclopedia of mobile phone behavior (pp. 591-606). Hershey, Pa: IGI Global.

Megan, A. W., William, M. B., \& Lis, E. (2016). The digital self: How social media serves as a setting that shapes youth's emotional experiences. Adolescent Research Review, 1(2), 163-173.

Miller, L., \& Lu, W. (2018, August 20). Gen Z is set to outnumber millennials. Bloomberg Economic. Retrieved from https://www.bloomberg.com/news/articles/2018-08-20/gen-z-to-outnumber-millennials-within-a-year-demographic-trends

Mitchell, A., \& Gottfried, J. (2015). Millennials and political news: Social media-the local TV for the next generation? Pew Research Center Journalism and Media. Retrieved from https://www.journalism.org/2015/06/01/millennials-political-news/

Montag, P. W. (2016). Carpe diem instead of losing your social mind: Beyond digital addiction and why we all suffer from digital overuse. Cogent Psychology, 3(1), 1157281.

Moreno, M. A., Jelenchick, L. A., Egan, K. G., Cox, E., Young, H., Gannon, K. E., \& Becker, T. (2011). Feeling bad on Facebook: Depression disclosures by college students on a social networking site. Depression and Anxiety, 28(6), 447-455.

Muise, A., Christofides, E., \& Desmarais, S. (2009). More information than you ever wanted: Does Facebook bring out the green-eyed monster of jealousy? Cyberpsychology \& Behavior, 12(4), 441-444.

Müller, K. W., Dreier, M., Duven, E., Giralt, S., Beutel, M. E., \& Wölfling, K. A. (2016). Hidden type of Internet Addiction? Intense and addictive use of social networking sites in adolescents. Computers in Human Behavior, 55, 172-177.

Nazir, S., \& Samaha, M. (2016). Relationships among smartphone addiction, anxiety, and family relations. Behavior \& Information Technology, 36(10), 1046-1052.

Oberst, U., Wegmann, E., Stodt, B., Brand, M., \& Chamarro, A. (2017). Negative consequences from heavy social networking in adolescents: The mediating role of fear of missing out. Journal of Adolescence, 55, 51-60.

Przybylski, A. K., Murayama, K., DeHaan, C. R., \& Gladwell, V. (2013). Motivational, emotional, and behavioral correlates of fear of missing out. Computers in Human Behavior, 29, 1841-1848.

Rosengard, D., Tucker-McLaughlin, M., \& Brown, T. (2014). Students and social news: How college students share news through social media. Electronic News. Retrieved from https://journals.sagepub.com/doi/10.1177/1931243114546448

Schimmenti, A., Passanisi, A., Gervasi, A. M., Manzella, S., \& Fama, F. I. (2015). Insecure attachment attitudes in the onset of problematic Internet use among late adolescents. Childpsychiatry Human Development, 45, 588-595.

Schou, A. C., \& Pallesen, S. (2014). Social network site addiction-an overview. Current Pharmaceutical Design, 20 (25), $4053-4061$.

Seemiller, C., \& Grace, M. (2016). Generation Z goes to college. San Francisco, California: Jossey-Brass.

Smith, A., \& Monica, A. (2016). Social media use in 2016. Washington, DC: Pew Research Center Internet \& Technology.

Song, I., LaRose, R., Eastin, M. S., \& Lin, C. A. (2004). Internet gratifications and internet addiction: On the uses and abuses of new media. Cyberpsychology and Behavior, 7, 384-394.

Statista Facts on Social Networks. (2015). Average numbers of hours per day spent by social media users on all social media channels. Retrieved from https://www.statista.com/statistics/270229/usage-duration-of-social networks-by-country/

Statista Facts on Social Networks. (2016). Social Media. Retrieved from https://www.statista.com/topics/1164/social-networks/

Tandoc, Jr. E., \& Johnson, E. (2016). Most students get breaking news first from Twitter. Newspaper Research Journal. Retrieved from https://journals.sagepub.com/doi/abs/10.1177/0739532916648961

Tsitsika, A. K., Tzavela, E. C., Janikian, M., Ólafsson, K., Iordache, A., Schoenmakers, T. M., Tzavara, C., \& Richardson, C. (2014). Online social networking in adolescence: Patterns of use in six European countries and links with psychosocial functioning. Journal of Adolescent Health, 55, 141-147.

Wang, C. W., Ho, R. T. H., Chan, C. L. W., \& Tse, S. (2015). Exploring personality characteristics of Chinese adolescents with internet-related addictive behaviors: Trait differences for gaming addiction and social networking addiction. Addictive Behavior, 42, 32-35.

Wood, S. (2013). Generation Z as consumers: Trends and innovation. Institute for Emerging Issues. N.C. State University.

Wu, A. M. S., Cheung, V. I., Ku, L., \& Hung, E. P. W. (2013). Psychological risk factors of addiction to social networking sites among Chinese smartphone users. Journal of Behavioral Addictions, 2(3), 160-166. 